\title{
Difficulty of cooperation in iterated multi-Prisoner's Dilemma situation-Experimental evidence-1
}

Kazuhito Ogawa ${ }^{1}$, Tetsuya Kawamura ${ }^{2}$, Tatsuya Kikutani ${ }^{2}$, and Sobei H. Oda ${ }^{3}$

${ }^{1}$ Hiroshima City University, kz-ogawa@intl.hiroshima-cu.ac.jp,

${ }^{2}$ Kyoto University, ${ }^{3}$ Kyoto Sangyo University

\section{Introduction}

It is popular that two players face with each other repeatedly in different phases [1]. Workers usually have relationship with their colleagues not only at work but also privately. Governments make negotiations on agricultural trade in addition to industrial trade. A hotel chain competes with the rival in various areas. Do these situations facilitate cooperation? What kind of decision makings should players

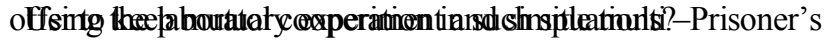
dilemma, we explore the effect of the change in the number of contacts and in the payoff structure, and then the characteristics of the strategy that leads players to reach mutual cooperation. When the number of contacts increases from one to two, players face with the more complicated situation and their behavior may be different from the single contact. The change of payoff structure will also affect their behavior. In such a case, finding the decision making to facilitate cooperation may be a hint for solving real problems.

\section{Theory and experiments on multi}

\section{contact}

In the context of the economic theory, [2], [6], and [7] use iterated prisoner's dilemma and examine multi-contact. First, [2] assume that two players play multi Iterated Prisoner's Dilemma (IPD) on game A and B simultaneously (Table2). They show that the discount factor is smaller in multi games than in a single game (B). Playing two identical games, say two B, does not facilitate cooperation.

Second, [7] and [8] assumes that a player has a strict concave utility function instead of linear function and focuses the repeated play of two identical games repeatedly. He proved that multimarket contact could facilitate cooperation even in sudher chrocterize multi contact from the discount factor.

However, the theoretical simplification lacks two factors which may affeet real decision making. First, as the situation changes

1 This research was supported by the Open Research

teaching economics and the research on its impact on society", Kyoto Sangyo University, Foundation for the Fusion of Science and Technology (FOST, http://www.fost.or.jp/) and JSPS research fellowship. decision making. First, as the situation changes from single to multi contact, the decision making set expands. Second, instead of two identical games, two different games change the payoff structure. This will influence player's behavior.

The theory is difficult to examine the effects of the factors, since focuses on the characteristics of the equilibrium and ignores the effect of the increase in the number of alternatives. It also focuses on the payoff structure change from the discount factor. However, the real effect of this change is untkeannducted a series of multi-IPD experiments and investigated the effects of two factors on human behavior. Although there are some experimental studies ([4], [5]) on multi contact, none of them check these factors seriously. Additionally, we sought for the behavior that leads to mutual cooperation. The behavior was mainly consisted of two kinds of Tit For Tat strategies and commonly observed among multi contact treatments.

\section{Experimental design}

\begin{tabular}{|l|l|l|}
\hline treatme & $\# \quad$ of & $\# \quad$ of \\
\hline A & 3Qbjects & Bounds \\
\hline B & 42 & 116 \\
\hline BB & 34 & 84 \\
\hline BBB & 18 & 78 \\
\hline AB & 40 & 116 \\
\hline BB' & 26 & 92 \\
\hline
\end{tabular}

Table 1: Profiles of experiments

\begin{tabular}{|c|c|c|c|c|c|c|c|c|}
\hline game A & D & D & game $B$ & C & D & game B' & C & D \\
\hline $\mathrm{C}$ & 800,800 & 0,1000 & $C$ & 800,800 & 0,1000 & $\mathrm{C}$ & 780,780 & 0,1000 \\
\hline D & 1000,0 & 210,210 & D & 1000,0 & 350,350 & D & 1000,0 & 260,260 \\
\hline
\end{tabular}

Table2: matrix A, B and B' in the left and right, respectively

Let us explain the experimental procedure. We have already conducted six treatments as in Table 1. Profiles of the treatments are shown in Table 1. The difference in the number of rounds comes from time constraint. All the treatments were done with Z-tree [5] in Kyoto Experimental Economics

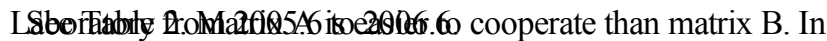
a series of experiments, the treatment lasted more than seventy rounds (Table 1). B' is almost as cooperative as B. In the 
(Table 1). B' is almost as cooperative as B. In the experiment, the subjects were not informed the number of rounds. The subjects played a game with the identical and anonymous opponent throughout the treatment. In single $\mathrm{A}$ and $\mathrm{B}$ treatment, in each round, they offer one of two alternatives. In multi contact treatment, in each round, they offer an alternative in each matrix, two or three alternatives in total. After choosing alternative(s), they observe their own choice(s), their opponent's choice(s), their own payoff, and their opponent's payoff. Finally, after the experiment, the monetary reward was paid according to the performance of a subject during the treatment. The monetary reward was paid in proportion to the total payoff with the showing yp feew

\begin{tabular}{|c|c|c|}
\hline 2 channels & B & hA \\
\hline 3 channels & BB & -- \\
\hline 4 channels & BBB, & AB \\
\hline
\end{tabular}

Table3: Experimental design

Row of the Table 3 means the predicted level of cooperation. Theoretically, B is more difficult to cooperate than A, and B' has almost the same level of cooperation as B.

Columns of the table indicate the number of the channels. Players in $\mathrm{B}$ and $\mathrm{A}$ have two channels, that is $\mathrm{C}$ and $\mathrm{D}$. Those in $\mathrm{BB}$ have three channels, $\mathrm{CC}, \mathrm{DD}$, and $\mathrm{CD}$. Those in $\mathrm{BBB}$ have four channels, CCC, DDD, CDD, and CCD. Those in $\mathrm{BB}^{\prime}$ and $\mathrm{AB}$ have four channels, $\mathrm{CC}, \mathrm{DD}, \mathrm{C}_{\mathrm{A}\left(\mathrm{B}^{\prime}\right)} \mathrm{DB}_{\mathrm{B}}$, and $\mathrm{D}_{\mathrm{A}\left(\mathrm{B}^{\prime}\right)}$ $\mathrm{C}_{B}$. The number of channels is different from the number of choices. A channel describes a substantial combination of choices that the subjects can offer to the opponent. Thus, in multi contact, the number of channels is more than in single contact. This may influence the subjects' behavior.

\section{Experimental Result}

$4-1$. The difference among treatments

\begin{tabular}{c|ccccc} 
average & All Rounds & 1-78 rounds & first half & second half & last 30 rounds \\
\hline Single B & 0.682 & 0.663 & 0.653 & 0.711 & 0.711 \\
Single A & 0.732 & 0.732 & 0.715 & 0.750 & 0.754 \\
MMT-BB & 0.615 & 0.612 & 0.571 & 0.659 & 0.671 \\
MMT-AB & 0.617 & 0.573 & 0.545 & 0.689 & 0.713 \\
MMT-BBB & 0.581 & 0.581 & 0.544 & 0.617 & 0.623 \\
MMT-BB' & 0.488 & 0.482 & 0.471 & 0.507 & 0.517
\end{tabular}

Table 4: Percent cooperation

\begin{tabular}{c|cccccc} 
& $\mathrm{A}$ & $\mathrm{B}$ & $\mathrm{AB}$ & $\mathrm{BB}$ & $\mathrm{BBB}$ & BB' $^{\prime}$ \\
\hline Variance & 0.00066 & 0.00504 & 0.00039 & 0.00064 & 0.00195 & 0.00224
\end{tabular}

Table 5: The variance of percent cooperation in the last 30 rounds

Let us explain the experimental result. First, we investigate the difference between the percent cooperation among treatments. Two-way ANOVA rejects the null hypothesis "percent cooperation among treatments is the same". The multiple comparison shows the following percent cooperation order; $\mathrm{A} \geqq \mathrm{B} \geqq \mathrm{AB}>\mathrm{BB}>\mathrm{BBB}>\mathrm{BB}$, at $5 \%$ level. The treatment difference may affect behavior.
Second, we check the growth rate of percent cooperation. Two-way ANOVA did not reject the null hypothesis. The treatment difference does not affect the growth rate of percent cobprivedativen.check the variance of percent cooperation in the last 30 rounds. The variance difference among treatments is significant at $1 \%$ level (Table 5).

These results show the difference between single contact and multi contact and the difference within multi contact treancoenttsooperation is higher under single treatments than under multi treatments. However, the variance is higher under single treatments than under multi treatments. Therefore, under single treatments the subjects can cooperate and break cooperation easily. On the other hand, under multi treatments, it takes rounds for reaching mutual cooperation. But once it is realized, it is robust. This suggests that when the number of channels decreases from three or four to two, the decision

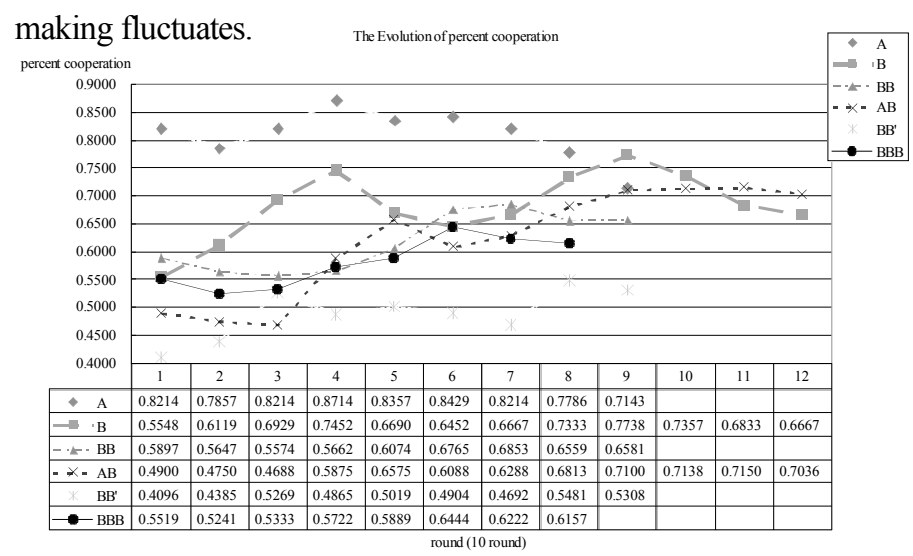

Figure 1: The evolution of percent cooperation.

In comparison within multi treatments, the treatment difference may affect the initial rounds decision makings. $A B$ is more difficult to cooperate than $\mathrm{BB}$ and $\mathrm{BBB}$ in the initial rdthadke (Feigperdent cooperation under $\mathrm{AB}$ is the largest. $\mathrm{AB}$ has two unique features; first, the number of channels is the largest and this makes cooperation difficult. Second, AB contains the matrix that facilitates cooperation. For decision makers in $\mathrm{AB}$, second feature is more powerful than first feature. Thus, regardless of four channels, $\mathrm{AB}$ realized high peradheamoperthionaniaheen $\mathrm{AB}$ is the smallest among the multi treatments. The existence of A decreases the fluctuation. The variance of $\mathrm{BB}$ is smaller than that of $\mathrm{BBB}$, although the evolution of percent cooperation is similar. This difference comes from the number of channels.

As for BB', B' is almost the same as B. However, percent cooperation remained low. As the result that the number of channels increases but that one of the matrices is replaced by the slightly different matrix, percent cooperation decreases. 
4-2.On the channel reduction and cooperation induction strategy (ChaRCIS)

Reaching and keeping mutual cooperation seem to be difficult under the multi treatments. However, keeping is easier under these treatments. This is a puzzle. Then, what does facilitate mutual cooperation under multi treatments?

This subsection explores the mechanism that facilitates and keeps mutual cooperation. It makes it clear that a subject has the clear-cut intention of cooperation but punishes the opponent severely when his or her opponent exploits him or her. It is adopted by most of the subjects in all the multi treatments. We call it "the channel reduction and cooperation

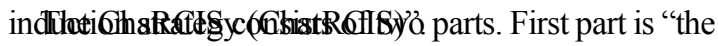
channel reduction", and second part is "the cooperation induction". The definition of the channel reduction is that in a game, a player chooses only a particular channel although he or she has more channels to choose. See Table5. A subject can use the channel reduction to send his or her opponent a message. For example, (CC)/ (CCC) appeals a cooperation signal. (DD)/ (DDD) appeals a signal of shirk or punishment. (CD)/ (CCD) and (DC)/ (DDC) are called the ambiguous choice since the interpretation depends on the opponent's previous choice. If the opponent offers (CC)/(CCC) ((DD)/ (DDD)) in the previous round, (CD)/ (CCD) and (DC)/ (DDC) havala signdil) $\varphi$ C dairk((copperation). (D(A) $\left.\mathrm{C}_{(\mathrm{B})}\right) /(\mathrm{CDD})(\mathrm{DD}) /(\mathrm{DDD})$

\begin{tabular}{c|cccc} 
BB & 0.523 & 0.101 & 0.083 & 0.293 \\
AB & 0.559 & 0.077 & 0.039 & 0.325 \\
BB' & 0.429 & 0.054 & 0.066 & 0.451 \\
BBB & 0.508 & 0.056 & 0.108 & 0.329
\end{tabular}

Table5: The percentages of channels in the all rounds.

Table 5 shows that most of the decision makings are in (CC)/ (CCC) or (DD)/ (DDD). Especially, this trend is more suitable in $\mathrm{AB}$ and $\mathrm{BB}$ '. In $\mathrm{BB}$ and $\mathrm{BBB}$, where all the matrices are the same, the percentage of the ambiguous channels is a little large. Calculating the coefficient of correlation between percent cooperation of a subject and the variance of the decision making, we find that as percent cooperation increases, the variance of the decision makings decreases. The subjects with high percent cooperation tend to narrow their channels.

The channel reduction is not enough since in the prisoner's dilemma situation, unilateral cooperation causes exploitation by the opponent. The subject who keeps on offering (CC)/ (CCC) sends a cooperative message to his or her opponent. But he or she may be exploited by his or her opponent, too.

Therefore, to facilitate cooperation in the long run, a subject has to make his or her opponent offer the cooperative alternatives. When the opponent is kind and willing to choose them, the subject does not have any trouble for reaching mutual cooperation. However, his or her opponent is not always kind. Then, what should the opponent do to reach and keep mutual cooperation?
In addition to the channel reduction, a subject has to utilize "the cooperation induction". It consists of punishment and conditional and unconditional cooperation (UC). Punishment and conditional cooperation are expressed by Tit for Tat. UC is to offer (CC)/ (CCC) and/or ambiguous channels when the opponent offers (DD)/ (DDD) to send the subject's intent and

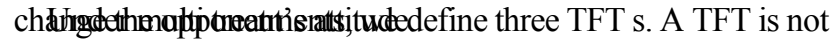
SPNE, but very popular strategy in human behavior. Table 6 indicates three TFT $\mathrm{s}$. This Table shows that T-TFT is the most patient and that R-TFT is the strictest. Furthermore, the cooperation induction contains $\mathrm{UC}$ although the percentage is small.

\begin{tabular}{|c|c|c|c|}
\hline The opponent's previous & T-TFT & R-TFT & S-TFT \\
\hline (affent)/ (CCC) & $(\mathrm{CC}) /$ & $(\mathrm{CC}) /$ & $(\mathrm{CC}) /$ \\
\hline (CD)/(CCD) & (CCC) & (DDG) & $(\mathrm{CD} g)$ \\
\hline (DC)/ (CDD) & $(\mathrm{CCC})$ & (DDD) & $(D C D)$ \\
\hline (DD)/(DDD) & (DDG) & (DDD) & (ODD) \\
\hline
\end{tabular}

Table 6: 3 TFT strategies (DDD) (DDD) (DDD)

The subject with ChaRCIS uses UC effectively. Typically, UC is used continuously in some rounds in two ways: it is used when subject $A$ sometimes exploits subject $B$ and subject $B$ hopes mutual cooperation. It is used by at lease one of the players to stem betrayal of both players. In the sense that UC can change the situation, it has the situation reset effect. In case that it fails to facilitate mutual cooperation, punishment is invoked. experiments, most of the subjects with high percent cooperation use the ChaRCIS. See Tables 7: in multi treatments, they used T-TFT, R-TFT and UC and lead their opponent to offer the cooperative choices. If they change the opponent's attitude, percent cooperation rises. However, a part of the ChaRCIS is vulnerable to the deviation of the opponent and it does not rise The ChaRCIS has to contain a punishment devichanethestypital cases. When the opponent is up for mutual cooperation, the subject tends to use T-TFT and UC to admit accidental mistakes. When the opponent is up for exploitation but the subject hope mutual cooperation, the subject utilizes R-TFT and UC to quit the circle of mutual deviation.

\section{Discussion}

We interpret prior experimental studies from the viewpoint of our results. Prior studies assert that multi contact basically facilitates cooperation. This is the opposite result with ours. However, taking it into account that their subjects might invoke the ChaRCIS, percent cooperation in the prior studies would be high. Additionally, they focus on the same payoff matrix from single and multi treatments. This is insufficient

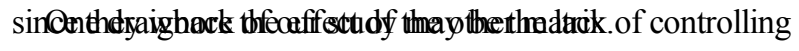
the discount factor. The discount factor control precludes subjects' long run behavior. In the discount factor control treatments, we will not attain the ChaRCIS. However, when we validate the multi contact strictly from the viewpoint of 
contact strictly from the viewpoint of infinitely iterated game theory, the control of the discount factor is necessary [3].

There is "Goal Expectation Theory (GET)" in experimental social psychology [10]. It characterizes the conditions to reaching mutual cooperation in Prisoner's Dilemma game.

Our subject who was not exploited by the opponent and whose percent cooperation was high was interpreted as acting according to GET. However, our experimental setting is more difficult than the ordinal PD games. To apply GET into the multi contact situation, the ChaRCIS will be important.

Finally, the ChaRCIS has relation with the GRIT strategy

[9]. GRIT was developed in the middle of the cold war era to realize a gradual de-escalation process in which one side makes a unilateral compromise with the hope that the opponent will do the same. This starts a positive spiral to mutual cooperation. It contains the effective reaction to the unilateral exploitation. Although it does not assume a PD

\section{Concluding Remarks}

We examined the multi contact effect on the behavior and attained the following result. First, the increase in the number of channels decreases percent cooperation. When the number of channels is four, the introduction of the easily cooperative game facilitates mutual cooperation. Second, the growth rate of percent cooperation is not significantly different among the treatments. These results indicate that the multi contact affects the decision makings initially but that it does not affect significantly those later. Third, the ChaRCIS is effective for the increase in the mutual cooperation. This may be helpful for

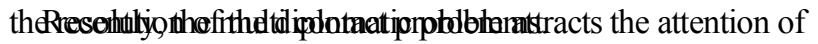
social psychology and mathematical biology as economics and a promising field. In the future work, we will conduct multi contact experiments to control the discount factor severely. Incorporating our future work and the present work, we hope to elucidate the effect of multi contact on human

\begin{tabular}{|c|c|c|c|c|c|c|c|c|}
\hline behan & jject \# & T-TFT & S-TFT & R-TFT & $\begin{array}{c}\text { BB } \\
\text { unconditional } \mathrm{C}\end{array}$ & unconditional D & others & $\%$ cooperation \\
\hline 7 & 13 & & $98.80 \%$ & & $1.20 \%$ & $0.00 \%$ & $0.00 \%$ & $99.40 \%$ \\
\hline & 14 & $100.00 \%$ & $0.00 \%$ & $0.00 \%$ & $0.00 \%$ & $0.00 \%$ & $0.00 \%$ & $98.81 \%$ \\
\hline & 15 & $75.90 \%$ & $0.00 \%$ & $20.48 \%$ & $2.41 \%$ & $1.20 \%$ & $0.00 \%$ & $95.24 \%$ \\
\hline 8 & 16 & $0.00 \%$ & $0.00 \%$ & $95.18 \%$ & $2.41 \%$ & $2.41 \%$ & $0.00 \%$ & $93.45 \%$ \\
\hline 10 & 1 & & $100.00 \%$ & & $0.00 \%$ & $0.00 \%$ & $0.00 \%$ & $100.00 \%$ \\
\hline 10 & 2 & & $100.00 \%$ & & $0.00 \%$ & $0.00 \%$ & $0.00 \%$ & $100.00 \%$ \\
\hline & 3 & $77.11 \%$ & $3.61 \%$ & $13.25 \%$ & $2.41 \%$ & $3.61 \%$ & $0.00 \%$ & $82.74 \%$ \\
\hline 11 & 4 & $80.72 \%$ & $10.84 \%$ & $0.00 \%$ & $7.23 \%$ & $1.20 \%$ & $0.00 \%$ & $85.71 \%$ \\
\hline & 9 & & $90.36 \%$ & & $4.82 \%$ & $4.82 \%$ & $0.00 \%$ & $94.05 \%$ \\
\hline 14 & 10 & & $97.59 \%$ & & $2.41 \%$ & $0.00 \%$ & $0.00 \%$ & $95.24 \%$ \\
\hline & 11 & & $100.00 \%$ & & $0.00 \%$ & $0.00 \%$ & $0.00 \%$ & $100.00 \%$ \\
\hline 15 & 12 & & $100.00 \%$ & & $0.00 \%$ & $0.00 \%$ & $0.00 \%$ & $100.00 \%$ \\
\hline & 13 & $80.72 \%$ & $0.00 \%$ & $16.87 \%$ & $1.20 \%$ & $1.20 \%$ & $0.00 \%$ & $98.21 \%$ \\
\hline 16 & 14 & $93.98 \%$ & $0.00 \%$ & $0.00 \%$ & $1.20 \%$ & $4.82 \%$ & $0.00 \%$ & $97.02 \%$ \\
\hline & 15 & $39.76 \%$ & $55.42 \%$ & $0.00 \%$ & $4.82 \%$ & $0.00 \%$ & $0.00 \%$ & $86.90 \%$ \\
\hline 17 & 16 & $46.99 \%$ & $0.00 \%$ & $30.12 \%$ & $6.02 \%$ & $16.87 \%$ & $0.00 \%$ & $76.19 \%$ \\
\hline
\end{tabular}

\begin{tabular}{|c|c|c|c|c|c|c|c|c|}
\hline \multicolumn{9}{|c|}{ BBB } \\
\hline Pair H & $\frac{\frac{3 u p j e c t ~ \#}{7}}{7}$ & $|-|+\mid$ & $\frac{\text { S-TFT }}{100.00 \%}$ & R-TFT & $\frac{\text { unconditional C }}{0.00 \%}$ & $\frac{\text { unconditional D }}{0.00 \%}$ & others & $\frac{\% \text { cooperation }}{100.00 \%}$ \\
\hline 4 & 8 & & $100.00 \%$ & & $0.00 \%$ & $0.00 \%$ & $0.00 \%$ & $100.00 \%$ \\
\hline & 13 & $70.13 \%$ & $1.30 \%$ & $10.39 \%$ & $7.79 \%$ & $7.79 \%$ & $2.60 \%$ & $73.08 \%$ \\
\hline 1 & 14 & $70.13 \%$ & $11.69 \%$ & $7.79 \%$ & $9.09 \%$ & $0.00 \%$ & $1.30 \%$ & $76.07 \%$ \\
\hline & 15 & & $100.00 \%$ & & $0.00 \%$ & $0.00 \%$ & $0.00 \%$ & $100.00 \%$ \\
\hline 0 & 16 & & $100.00 \%$ & & $0.00 \%$ & $0.00 \%$ & $0.00 \%$ & $100.00 \%$ \\
\hline & 17 & $84.42 \%$ & $0.00 \%$ & $5.19 \%$ & $6.49 \%$ & $2.60 \%$ & $1.30 \%$ & $91.45 \%$ \\
\hline & 18 & $0.00 \%$ & $89.61 \%$ & $0.00 \%$ & $2.60 \%$ & $7.79 \%$ & $0.00 \%$ & $87.18 \%$ \\
\hline
\end{tabular}

\begin{tabular}{|c|c|c|c|c|c|c|c|c|}
\hline Pair \# & Subject \# & T-TFT & S-TFT & R-TFT & $\begin{array}{l}\mathrm{BB}^{\prime} \\
\text { unconditional C }\end{array}$ & unconditional D & others & $\%$ cooperation \\
\hline \multirow[b]{2}{*}{1} & 1 & $0.00 \%$ & $98.90 \%$ & $0.00 \%$ & $0.00 \%$ & $1.10 \%$ & $0.00 \%$ & $85.87 \%$ \\
\hline & 2 & $85.71 \%$ & $12.09 \%$ & $0.00 \%$ & $2.20 \%$ & $0.00 \%$ & $0.00 \%$ & $\begin{array}{l}88.04 \% \\
\end{array}$ \\
\hline \multirow[t]{2}{*}{3} & 5 & $100.00 \%$ & $0.00 \%$ & $0.00 \%$ & $0.00 \%$ & $0.00 \%$ & $0.00 \%$ & $100.00 \%$ \\
\hline & $\begin{array}{l}0 \\
7\end{array}$ & $1.10 \%$ & $\begin{array}{l}100.00 \% \\
95.60 \%\end{array}$ & $0.00 \%$ & $\begin{array}{l}0.00 \% \\
330 \%\end{array}$ & $\begin{array}{l}0.00 \% \\
0.00 \%\end{array}$ & $\begin{array}{l}0.00 \% \\
0.00 \%\end{array}$ & $\begin{array}{l}99.46 \% \\
9239 \%\end{array}$ \\
\hline 4 & 8 & $0.00 \%$ & $100.00 \%$ & $0.00 \%$ & $0.00 \%$ & $0.00 \%$ & $0.00 \%$ & $91.30 \%$ \\
\hline \multirow{2}{*}{8} & 15 & & $89.01 \%$ & & $5.49 \%$ & $5.49 \%$ & $0.00 \%$ & $82.07 \%$ \\
\hline & 16 & $84.62 \%$ & $0.00 \%$ & $8.79 \%$ & $4.40 \%$ & $2.20 \%$ & $0.00 \%$ & $83.70 \%$ \\
\hline \multirow[t]{2}{*}{10} & 19 & $0.00 \%$ & $0.00 \%$ & $96.70 \%$ & $2.20 \%$ & $1.10 \%$ & $0.00 \%$ & $94.02 \%$ \\
\hline & 20 & $97.80 \%$ & $0.00 \%$ & $0.00 \%$ & $1.10 \%$ & $1.10 \%$ & $0.00 \%$ & $95.11 \%$ \\
\hline
\end{tabular}

\begin{tabular}{|c|c|c|c|c|c|c|c|c|}
\hline \multicolumn{9}{|c|}{$A B$} \\
\hline Pair \# & Subject \# & T-TFT & S-TFT & R-TFT & unconditional C & unconditional D & others & $\%$ cooperation \\
\hline & & $7.83 \%$ & $8.70 \%$ & $71.30 \%$ & $1.74 \%$ & $8.70 \%$ & $1.74 \%$ & $73.71 \%$ \\
\hline 1 & 2 & $69.57 \%$ & $5.22 \%$ & $14.78 \%$ & $8.70 \%$ & $1.74 \%$ & $0.00 \%$ & $78.88 \%$ \\
\hline & 3 & $22.61 \%$ & $0.00 \%$ & $73.91 \%$ & $1.74 \%$ & $1.74 \%$ & $0.00 \%$ & $96.98 \%$ \\
\hline 2 & 4 & $0.00 \%$ & $0.00 \%$ & $94.78 \%$ & $1.74 \%$ & $3.48 \%$ & $0.00 \%$ & $96.12 \%$ \\
\hline & 9 & $8.70 \%$ & $10.43 \%$ & $7304 \%$ & $5.22 \%$ & $261 \%$ & $0.00 \%$ & $81.47 \%$ \\
\hline 5 & 10 & $1478 \%$ & $7130 \%$ & $261 \%$ & $522 \%$ & $609 \%$ & $000 \%$ & $8017 \%$ \\
\hline & 11 & $\begin{array}{l}1.00 \% \\
0.00 \%\end{array}$ & $0.00 \%$ & $\begin{array}{l}99.13 \% \\
9.61 \%\end{array}$ & $0.87 \%$ & $\begin{array}{l}0.00 \% \\
0.09 \%\end{array}$ & $0.00 \%$ & $\begin{array}{l}98.28 \% \\
9.17 \%\end{array}$ \\
\hline 6 & 12 & & $99.13 \%$ & & $0.87 \%$ & $0.00 \%$ & $0.00 \%$ & $98.71 \%$ \\
\hline 10 & 19 & & $100.00 \%$ & & $0.00 \%$ & $0.00 \%$ & $0.00 \%$ & $99.57 \%$ \\
\hline & 20 & $100.00 \%$ & $0.00 \%$ & $0.00 \%$ & $0.00 \%$ & $0.00 \%$ & $0.00 \%$ & $100.00 \%$ \\
\hline & 1 & $88.70 \%$ & $2.61 \%$ & $3.48 \%$ & $2.61 \%$ & $1.74 \%$ & $0.87 \%$ & $92.67 \%$ \\
\hline 12 & 2 & $92.17 \%$ & $0.00 \%$ & $2.61 \%$ & $1.74 \%$ & $1.74 \%$ & $1.74 \%$ & $92.67 \%$ \\
\hline & 5 & & $100.00 \%$ & & $0.00 \%$ & $0.00 \%$ & $0.00 \%$ & $100.00 \%$ \\
\hline & 6 & & $100.00 \%$ & & $0.00 \%$ & $0.00 \%$ & $0.00 \%$ & $100.00 \%$ \\
\hline & 9 & $0.00 \%$ & $0.00 \%$ & $98.26 \%$ & $0.87 \%$ & $0.87 \%$ & $0.00 \%$ & $72.84 \%$ \\
\hline 16 & 10 & $0.00 \%$ & $0.00 \%$ & $93.91 \%$ & $5.22 \%$ & $0.87 \%$ & $0.00 \%$ & $74.57 \%$ \\
\hline & 11 & $5.22 \%$ & $4.35 \%$ & $85.22 \%$ & $3.48 \%$ & $0.87 \%$ & $0.87 \%$ & $85.34 \%$ \\
\hline 17 & 12 & $\begin{array}{l}0.87 \% \\
0.87 \%\end{array}$ & $\begin{array}{l}4.601 \% \\
2.61 \%\end{array}$ & $\begin{array}{l}82.61 \% \\
82.60\end{array}$ & $\begin{array}{l}0.96 \% \\
6.96 \%\end{array}$ & $6.96 \%$ & $\begin{array}{l}0.00 \% \\
0.0 \%\end{array}$ & $\begin{array}{l}83.19 \% \\
83.4 \%\end{array}$ \\
\hline & 13 & $93.04 \%$ & $0.00 \%$ & $0.00 \%$ & $1.74 \%$ & $5.22 \%$ & $0.00 \%$ & $93.53 \%$ \\
\hline & 14 & $91.30 \%$ & $0.00 \%$ & $0.00 \%$ & $4.35 \%$ & $4.35 \%$ & $0.00 \%$ & $96.55 \%$ \\
\hline & 17 & $76.52 \%$ & $0.00 \%$ & $7.83 \%$ & $14.78 \%$ & $0.00 \%$ & $0.87 \%$ & $90.52 \%$ \\
\hline 20 & 18 & $0.00 \%$ & $75.65 \%$ & $0.00 \%$ & $2.61 \%$ & $20.87 \%$ & $0.87 \%$ & $74.14 \%$ \\
\hline
\end{tabular}

\begin{tabular}{|c|c|c|c|c|c|c|c|c|}
\hline \multirow{3}{*}{ Pair \# } & \multicolumn{8}{|c|}{ BB } \\
\hline & Subject \# & T-TFT & S-TFT & R-TFT & unconditional C & unconditional D & others & $\%$ cooperation \\
\hline & $\begin{array}{l}13 \\
14\end{array}$ & $10000 \%$ & $\begin{array}{l}98.80 \% \\
000 \%\end{array}$ & $000 \%$ & $1.20 \%$ & $\begin{array}{l}0.00 \% \\
0.00 \%\end{array}$ & $\begin{array}{l}0.00 \% \\
0.00 \%\end{array}$ & $\begin{array}{l}99.40 \% \\
98.81 \%\end{array}$ \\
\hline \multirow{2}{*}{8} & $\begin{array}{l}14 \\
15\end{array}$ & $75.90 \%$ & $\begin{array}{l}0.00 \% \\
0.00 \%\end{array}$ & $20.48 \%$ & $\begin{array}{l}0.00 \% \\
241 \%\end{array}$ & $\begin{array}{l}0.00 \% \\
1.20 \%\end{array}$ & $\begin{array}{l}0.00 \% \\
0.00 \%\end{array}$ & $\begin{array}{l}98.81 \% \\
95.24 \%\end{array}$ \\
\hline & 16 & $0.00 \%$ & $0.00 \%$ & $95.18 \%$ & $241 \%$ & $241 \%$ & $0.00 \%$ & $93.45 \%$ \\
\hline \multirow{2}{*}{10} & 1 & & $100.00 \%$ & & $0.00 \%$ & $0.00 \%$ & $0.00 \%$ & $100.00 \%$ \\
\hline & 2 & & $100.00 \%$ & & $0.00 \%$ & $0.00 \%$ & $0.00 \%$ & $100.00 \%$ \\
\hline \multirow{2}{*}{11} & 3 & $77.11 \%$ & $3.61 \%$ & $13.25 \%$ & $2.41 \%$ & $3.61 \%$ & $0.00 \%$ & $82.74 \%$ \\
\hline & 4 & $80.72 \%$ & $10.84 \%$ & $0.00 \%$ & $7.23 \%$ & $120 \%$ & $0.00 \%$ & $8571 \%$ \\
\hline \multirow{2}{*}{14} & 9 & & $90.36 \%$ & & $4.82 \%$ & $4.82 \%$ & $0.00 \%$ & $94.05 \%$ \\
\hline & 10 & & $97.59 \%$ & & $2.41 \%$ & $0.00 \%$ & $0.00 \%$ & $95.24 \%$ \\
\hline \multirow{2}{*}{15} & 11 & & $100.00 \%$ & & $0.00 \%$ & $0.00 \%$ & $0.00 \%$ & $100.00 \%$ \\
\hline & 12 & & $100.00 \%$ & & $0.00 \%$ & $0.00 \%$ & $0.00 \%$ & $100.00 \%$ \\
\hline \multirow{2}{*}{16} & 13 & $80.72 \%$ & $0.00 \%$ & $16.87 \%$ & $1.20 \%$ & $1.20 \%$ & $0.00 \%$ & $98.21 \%$ \\
\hline & 14 & $93.98 \%$ & $0.00 \%$ & $0.00 \%$ & $1.20 \%$ & $4.82 \%$ & $0.00 \%$ & $97.02 \%$ \\
\hline \multirow[b]{2}{*}{17} & & $39.76 \%$ & $55.42 \%$ & $0.00 \%$ & $4.82 \%$ & $0.00 \%$ & $0.00 \%$ & $86.90 \%$ \\
\hline & $\begin{array}{l}15 \\
16\end{array}$ & $\begin{array}{l}39.16 \% \% \\
46.99 \%\end{array}$ & $\begin{array}{l}55.4 \% \% \\
0.00 \%\end{array}$ & $\begin{array}{l}0.00 \% \\
30.12 \%\end{array}$ & $\begin{array}{l}4.02 \% \\
6.02 \%\end{array}$ & $\begin{array}{l}.0 .07 \% \\
16.87 \%\end{array}$ & $0.00 \%$ & $76.19 \%$ \\
\hline
\end{tabular}

Table 7: decision makings by the cooperative subjects

\section{Reference}

1. Aoki, M., 2001, Toward a Comparative Institutional Analysis, MIT preßsernheim, B. D. and Whinston M. D. "Multimarket Contact and Collusive Behavior", Rand Journal of Economics, vol.21, 1990

3. Dal Bo, P., "Cooperation under the Shadow of the Future: Experimental Evidence from Infinitely Repeated Games", forthcoming in American Economic Review

4. Feinberg, R.M. and Sherman, R. "Mutual Forbearance under Experimental Conditions", Southern Economic Journal, vol.54, 1988

5. Fischbacher, U. "z-Tree - Zurich Toolbox for Readymade Economic Experiments -Experimenter's Manual", Working Paper, Institute for Empirical Research in Economics, University of Zurich, 699hillips, O. R. and Mason, C. F. "Mutual forbearance in experimental conglomerate markets", Rand Journal of Economics, Vol.Spadin,ol 89 G., "On Interdependent Supergames: Multimarket Contact, Concavity and Collusion", Journal of Economic Theory 89/1, \$98\$agnolo G., " Social Relations and Cooperation in Organizations", Journal of Economic Behavior and Organization 38/1, 1999.

9. Osgood, E.C., "Alternative to War or Surrender", Illinois University Press, 1962

10. Pruitt, D.G. and Kimmel, M.J., "Twenty years of experimental gaming: critique, synthesis, and suggestions for the future", Annual Review of Psychology, 1977 